\title{
UNIQUE CONTINUATION AT INFINITY OF SOLUTIONS TO SCHRÖDINGER EQUATIONS WITH COMPLEX-VALUED POTENTIALS
}

\author{
by J. CRUZ-SAMPEDRO* \\ (Received 13th March 1997)
}

Dedicated to the memory of Professor Olgierd A. Biberstein (1921-1997)

\begin{abstract}
We obtain optimal $L^{2}$-lower bounds for nonzero solutions to $-\Delta \Psi+V \Psi=E \Psi$ in $\mathbf{R}^{n}, n \geq 2, E \in \mathbf{R}$, where $V$ is a measurable complex-valued potential with $V(x)=O\left(|x|^{-c}\right)$ as $|x| \rightarrow \infty$, for some $\epsilon \in \mathbf{R}$. We show that if $3 \delta=\max \{0,1-2 \epsilon\}$ and $\exp \left(\tau|x|^{1+\delta}\right) \Psi \in L^{2}\left(\mathbf{R}^{n}\right)$ for all $\tau>0$, then $\Psi$ has compact support. This result is new for $0<\epsilon<1 / 2$ and generalizes similar results obtained by Meshkov for $\epsilon=0$, and by Froese, Herbst, M. Hoffmann-Ostenhof, and T. Hoffmann-Ostenhof for both $\epsilon \leq 0$ and $\epsilon \geq 1 / 2$. These $L^{2}$-lower bounds are well known to be optimal for $\epsilon \geq 1 / 2$ while for $\epsilon<1 / 2$ this last is only known for $\epsilon=0$ in view of an example of Meshkov. We generalize Meshkov's example for $\epsilon<1 / 2$ and thus show that for complex-valued potentials our result is optimal for all $\epsilon \in \mathbf{R}$.
\end{abstract}

1991 Mathematics subject classification: 35J10, 35B40, 35B60, $81 \mathrm{C} 05$.

\section{Introduction}

Let $\epsilon \in \mathbf{R}$ be given and suppose $V$ is a measurable complex-valued function on $\mathbf{R}^{n}$ that satisfies

$$
V(x)=O\left(|x|^{-\epsilon}\right), \quad \text { as } \quad|x| \rightarrow \infty
$$

In this paper we investigate the fastest possible rate of decay of the solutions to

$$
-\Delta \Psi+V \Psi=E \Psi
$$

on $\mathbf{R}^{n}$, where $\Delta$ is the Laplacian on $\mathbf{R}^{n}, n \geq 2$, and $E \in \mathbf{R}$. Without further assumptions on $V$ we prove:

Theorem 1. Let $V, E$, and $\epsilon$ be as in (1.1) and (1.2) and let $3 \delta=\max \{0,1-2 \epsilon\}$. Let $\Psi \in H_{\text {loc }}^{2}\left(\mathbf{R}^{n}\right)$ be a nonzero solution of (1.2) that satisfies

- Supported in part by CONACyT, Mexico. 


$$
\exp \left(\tau|x|^{1+\delta}\right) \Psi \in L^{2}\left(\mathbf{R}^{n}\right)
$$

for all $\tau>0$. Then $\Psi$ has compact support.

We view this theorem as a unique continuation at infinity result and prove it through a Carleman-like estimate that generalizes work of Meshkov [10] for $\epsilon=0$. A similar result for both $\epsilon \leq 0$ and $\epsilon \geq 1 / 2$ has been obtained using different methods by Froese, Herbst, T. Hoffmann-Ostenhof, and M. Hoffmann-Ostenhof [8]. It is well known that Theorem 1 is optimal for $\epsilon \geq 1 / 2$ while for $\epsilon<1 / 2$ this last is only known for $\epsilon=0$ in view of an example due to Meshkov [10]. We generalize Meshkov's example for $\epsilon<1 / 2$ and prove:

Theorem 2. Let $\epsilon<1 / 2$ and $\delta>0$ satisfy $2 \epsilon+3 \delta=1$. Then there exist a continuous complex-valued function $V$ on $\mathbf{R}^{2}$ satisfying (1.1), and a $C^{2}$-function $\Psi$ which does not have compact support and satisfies $\Delta \Psi=V \Psi$ on $\mathbf{R}^{2}$ and

$$
\Psi(x)=O\left(\exp \left(-\beta|x|^{1+\delta}\right)\right), \quad \text { as } \quad|x| \rightarrow \infty,
$$

for some $\beta>0$.

Thus, for complex-valued potentials Theorem 1 is optimal for all $\epsilon \in \mathbf{R}$.

The above results are closely related to the following question posed by $\mathbf{B}$. Simon [12]. Let $V$ be a real-valued potential and suppose that $\Psi \not \equiv 0$ satisfies $(-\Delta+V-E) \Psi=0$ in $\mathbf{R}^{n}$. Is it true that if $-\Delta+V$ does not have compact resolvent, then $\exp (\tau|x|) \Psi \notin L^{2}\left(\mathbf{R}^{n}\right)$ for $\tau>0$ sufficiently large? The answer to this question is not known but a positive response is suggested by the sharp exponential upper and lower bounds of different kinds already established for several classes of potentials $[1,2,3,4$, $5,6,7,8]$. The results for $0<\epsilon<1 / 2$ presented in this paper show that a proof of an affirmative answer to Simon's question has to use in an essential way the fact that $V$ is real-valued, even if $V(x)$ goes to zero as $|x|$ goes to infinity.

The general strategy to prove Theorems 1 and 2 is that of Meshkov [10]; however, the author also benefited from [11]. In Section 2 we obtain Carleman-like estimates near infinity and use these to prove Theorem 1. In Section 3 we generalize Meshkov's example.

\section{Carleman-like estimates in exterior domains}

Theorem 1 will be derived from the following Carleman-like estimate at infinity.

Lemma 2.1. Let $\rho>0, E \in \mathbf{R}$, and $\delta \geq 0$ be given. Set $\Omega_{\rho}=\left\{x \in \mathbf{R}^{n}:|x|>\rho\right\}$ and fix $\kappa$ such that $-3 \delta<\kappa \leq 2-\delta$. Then there exist a constant $K$ independent of $\tau$ and $\tau_{0}>0$ such that for any $v \in C_{0}^{\infty}\left(\Omega_{\rho}\right)$ and $\tau>\tau_{0}$ we have 


$$
\int_{\Omega_{\rho}}|x|^{3 \delta-n+\kappa}|v(x)|^{2} \exp \left(2 \tau|x|^{1+\delta}\right) d x \leq \frac{K}{\tau^{3}} \int_{\Omega_{\rho}}|x|^{\alpha+1-n}|(\Delta+E) v(x)|^{2} \exp \left(2 \tau|x|^{1+\delta}\right) d x .
$$

Remark. The constant $E$ in (2.1) is important only for $0 \leq \delta<1 / 3$.

Proof. Since $E \in \mathbf{R}$ we may assume without loss of generality that $v$ is real-valued. Set $r=|x|, \omega=x /|x|, \quad$ and $\alpha=1+\delta$. For $\tau>0$ set $u=\exp \left(\tau r^{a}\right) v$ and $\Delta_{\tau}=\exp \left(\tau r^{\alpha}\right) \Delta \exp \left(-\tau r^{\alpha}\right)$, where $\exp \left(\tau r^{\alpha}\right)$ and $\exp \left(-\tau r^{\alpha}\right)$ are multiplication operators. Using this notation we find that (2.1) is equivalent to

$$
\int r^{3 \delta+\kappa-1}|\boldsymbol{u}|^{2} d r d \omega \leq \frac{K}{\tau^{3}} \int r^{\kappa}\left|\left(\Delta_{\tau}+E\right) u\right|^{2} d r d \omega,
$$

where $d \omega$ denotes the Lebesgue measure on $S^{n-1}$. Defining $L=2 \tau \alpha r^{r-1}+\partial_{r}$ we have

$$
L+\Delta_{\tau}=\partial_{r r}+\tau^{2} \alpha^{2} r^{2 x-2}+\frac{n-1}{r} \partial_{r}-\tau \alpha(n+\alpha-2) r^{x^{2}-2}+\frac{1}{r^{2}} \Lambda,
$$

where $\Lambda$ is the Laplace-Beltrami operator on the unit sphere $S^{n-1}$. Setting $u_{r}=\partial_{r} u$ and integrating by parts with respect to $r$ we obtain

$$
\begin{aligned}
\int r^{\kappa}\left|\left(\Delta_{\tau}+E\right) u\right|^{2} d r d \omega \geq & \int r^{\kappa}\left(|L u|^{2}-2 L u\left(L+\Delta_{\tau}+E\right) u\right) d r d \omega \\
= & \int\left(4 \tau^{2} \alpha^{2} r^{2 \alpha+\kappa-2}+2 \tau \alpha(\alpha+\kappa-2 n+1) r^{\alpha+\kappa-2}\right) u_{r}^{2} d r d \omega \\
& +2 \tau \alpha \int\left[\left(\tau^{2} \alpha^{2}(3 \alpha+\kappa-3) r^{3 z+\kappa-4}\right.\right. \\
& \left.-\tau \alpha(2 \alpha-3+\kappa)(n+\alpha-2) r^{2 \alpha+\kappa-4}\right) u^{2} \\
& \left.+(\alpha+\kappa-3) r^{\alpha+\kappa-4} u \Lambda u-\alpha E(\alpha+\kappa-1) r^{\alpha+\kappa-2} u^{2}\right] d r d \omega \\
= & \int 2 \tau^{3} \alpha^{3}(3 \delta+\kappa) r^{3 \delta+\kappa-1} \\
& {\left[1-\frac{1}{\tau \alpha(3 \delta+\kappa)}\left(\frac{(2 \alpha+\kappa-3)(n+\alpha-2)}{r^{2}}+\frac{E(\delta+\kappa)}{\tau \alpha r^{2 \delta}}\right)\right] u^{2} d r d \omega } \\
& +\int 2 \tau \alpha\left[2 \tau \alpha r^{\alpha+2 \delta}\left(1+\frac{\alpha+\kappa-2 n+1}{2 \tau \alpha r^{2}}\right) u_{r}^{2}+(\delta+\kappa-2) r^{2+\kappa-4} u \Lambda u\right] d r d \omega .
\end{aligned}
$$

Since $-3 \delta<\kappa \leq 2-\delta$ and $\Lambda$ is a negative operator on $L^{2}\left(S^{n-1}, d \omega\right)$ we have

$$
\int r^{\kappa}\left|\left(\Delta_{\tau}+E\right) u\right|^{2} d r d \omega \geq \int 2 \tau^{3} \alpha^{3}(3 \delta+\kappa) r^{3 \delta+\kappa-1} u^{2}\left(1+O\left(\frac{1}{\tau}\right)\right) d r d \omega,
$$

from which (2.2) follows. 
Proof of Theorem 1. Theorem 1 follows from (2.1) using standard arguments that we sketch here for the sake of completeness. Let $V, E, \epsilon, \delta$ and $\Psi$ be as in Theorem 1 . Assuming that

$$
\int_{\mathbf{R}^{n}}|\Psi(x)|^{2} \exp \left(2 \tau|x|^{1+\delta}\right) d x<\infty
$$

for all $\tau>0$, we will prove that $\Psi$ has compact support. Using $L^{2}$-interior estimates [9], it follows from (2.3) that

$$
\int_{\mathbf{R}^{n}}\left|D^{\beta} \Psi(x)\right|^{2} \exp \left(2 \tau|x|^{1+\delta}\right) d x<\infty
$$

for all $\tau>0$ and all multi-indices $\beta$ such that $|\beta| \leq 2$. Let $\kappa$ be as in Lemma 2.1 and fix $\rho \geq 0$ so that $\left.|V(x \mid) \leq C| x\right|^{-\varepsilon}$ for $x \in \Omega_{\rho}$. A simple estimate, using (2.1) and $3 \delta=\max \{0,1-2 \epsilon\}$, shows that there exist a constant $K$ independent of $\tau$ and $\tau_{0}>0$ such that for any $v \in C_{0}^{\infty}\left(\Omega_{\rho}\right)$ and $\tau>\tau_{0}$ we have

$$
\int_{\Omega_{\rho}}|x|^{3 \delta-n+\kappa}|v(x)|^{2} \exp \left(2 \tau|x|^{1+\delta}\right) d x \leq \frac{K}{\tau^{3}} \int_{\Omega_{\rho}}|x|^{\kappa+1-n}|(\Delta-V+E) v(x)|^{2} \exp \left(2 \tau|x|^{1+\delta}\right) d x .
$$

Let $h$ be a $C^{\infty}$-function on $\mathbf{R}^{n}$ which takes values between 0 and 1 , vanishes on $|x| \leq \rho+1 / 2$, and equals 1 on $|x| \geq \rho+1$. Let $\phi \in C_{0}^{\infty}\left(\mathbf{R}^{n}\right)$ be a function which equals 1 on $|x| \leq 1$ and set $\phi_{\lambda}(x)=\phi(x / \lambda)$ for $\lambda>0$. An approximation argument shows that (2.5) holds for every $v \in H^{2}\left(\mathbf{R}^{n}\right)$ with compact support contained in $\Omega_{\rho}$. Hence (2.5) holds on $\Omega_{\rho}$ for $v_{\lambda}=\phi_{\lambda} \Psi h$ and therefore, using (2.4), for $v=\Psi h$. Since $\Psi$ satisfies (1.2) and $h(x)=1$ on $\Omega_{\rho+1}$ we obtain

$$
\int_{\Omega_{\rho+1}}|x|^{3 \delta-n+\kappa}|\Psi(x)|^{2} d x \leq \frac{K}{\tau^{3}} \int_{\rho \leq|x| \leq p+1}|x|^{\kappa+1-n}|(\Delta-V+E) v(x)|^{2} d x .
$$

Letting $\tau$ go to infinity in this last estimate we find that $\Psi \equiv 0$ on $\Omega_{\rho+1}$.

\section{Examples}

Although the essential idea of the construction given below is that of Meshkov [10], we present the details for the reader's convenience. For $\rho>0$ we will denote by $A(\alpha, \beta)$ the annulus in $\mathbf{R}^{2}$ defined by $\rho+\alpha \rho^{(1-\delta) / 2} \leq r \leq \rho+\beta \rho^{(1-\delta) / 2}$.

Lemma 3.1. Let $\delta>0$ and $\epsilon \in \mathbf{R}$ satisfy $2 \epsilon+3 \delta=1$. For a fixed and large $\rho>0$ let $n$ and $k$ be positive integers such that $\left|n-\rho^{1+\delta}\right| \leq 1$ and $\left|k-6(1+\delta) \rho^{(1+\delta) / 2}\right| \leq$ $1+20 \delta(1+\delta)$. Then there exist complex-valued functions $u$ and $V$ on $A(0,6)$ possessing the following properties: 
(a) The function $u$ is of class $C^{2}$ and satisfies

$$
\Delta u+V u=0 \quad \text { on } A(0,6)
$$

(b) There exists a constant $C$ independent of $\rho, n$, and $k$ such that

$$
|V(r, \theta)| \leq C r^{-\epsilon} \quad \text { on } A(0,6) \text {. }
$$

(c) For a constant $a>0$ we have

$$
u(r, \theta)= \begin{cases}r^{-n} \exp (-i n \theta) & \text { on } A(0,0.1) \\ a r^{-n-k} \exp i(-n-k) \theta & \text { on } A(5.9,6)\end{cases}
$$

Therefore $V \equiv 0$ on the annuli $A(0,0.1)$ and $A(5.9,6)$.

(d) Let $m(r)=\max \{|u(r, \theta)|, 0 \leq \theta \leq 2 \pi\}$. Then

$$
\log m(r)-\log m(\rho) \leq \log 2-\frac{1}{6} \int_{\rho}^{r} t^{\delta} d t
$$

for $\rho \leq r \leq \rho+6 \rho^{(1-\delta) / 2}$.

Proof of Theorem 2. First we fix a large $\rho_{1}>0$ and set $\rho_{j+1}=\rho_{j}+6 \rho_{j}^{(1-\delta) / 2}$ for $j=1,2, \ldots$ Then we set $n_{j}=\left[\rho_{j}^{1+\delta}\right]$, where $[x]=\max \{n \in \mathbf{Z}: n \leq x\}$, and $k_{j}=n_{j+1}-n_{j}$. For $j=1,2, \ldots$ we have $n_{j}=\rho_{j}^{1+\delta}-\gamma_{j}$, with $0 \leq \gamma_{j}<1$, and

$$
\begin{aligned}
k_{j} & =\rho_{j+1}^{1+\delta}-\rho_{j}^{1+\delta}+\gamma_{j}-\gamma_{j+1} \\
& =\rho_{j}^{1+\delta}\left(1+6 \rho_{j}^{-(1+\delta) / 2}\right)^{1+\delta}-\rho_{j}^{1+\delta}+\gamma_{j}-\gamma_{j+1} \\
& =6(1+\delta) \rho_{j}^{(1+\delta) / 2}+18 \delta(1+\delta)+O\left(\rho_{j}^{-(1+\delta) / 2}\right)+\gamma_{j}-\gamma_{j+1} .
\end{aligned}
$$

Therefore if $\rho_{1}$ is large we may assume that $\left|k_{j}-6(1+\delta) \rho_{j}^{(1+\delta) / 2}\right| \leq 1+20 \delta(1+\delta)$. For $j=1,2, \ldots$, let $a_{j}$ be constants and $u_{j}$ and $V_{j}$ be functions constructed on $\rho_{j} \leq r \leq \rho_{j+1}$ as in Lemma 3.1. Then $u_{j}\left(\rho_{j}, \theta\right)=\rho_{j}^{-n_{j}} \exp \left(-i n_{j} \theta\right)$ and $u_{j}\left(\rho_{j+1}, \theta\right)=a_{j} \rho_{j+1}^{-n_{j+1}} \exp \left(-i n_{j+1} \theta\right)$. Since $\rho_{j} \rightarrow \infty$ as $j \rightarrow \infty$, then for $r>\rho_{1}$ we set $V(r, \theta)=V_{j}(r, \theta)$ and $\Psi(r, \theta)=A_{j} u_{j}(r, \theta)$ for $\rho_{j} \leq r \leq \rho_{j+1}$, where we define $A_{j}=a_{0} a_{1} \ldots a_{j-1}$, for $j=1,2, \ldots$, and $a_{0}=1$. Clearly $V$ satisfies (1.2) and $\Psi$ is of class $C^{2}$ and satisfies $-\Delta \Psi+V \Psi=0$ on $\Omega_{\rho_{1}}$. To prove that $\Psi$ satisfies (1.4) we set $\mu(r)=\max \{|\Psi(r, \theta)|: 0 \leq \theta \leq 2 \pi\}$ for $r>\rho_{1}$ and pick $l$ such that $\rho_{l} \leq r \leq \rho_{l+1}$. Then

$$
\log \mu(r)=\left(\log m_{l}(r)-\log m_{l}\left(\rho_{l}\right)+\ldots+\left(\log m_{1}\left(\rho_{2}\right)-\log m_{1}\left(\rho_{1}\right)\right)+\log m_{1}\left(\rho_{1}\right),\right.
$$

where $m_{j}(r)$ is as in (d) of Lemma 3.1. Using (d) of this lemma we find that 


$$
\log \mu(r) \leq l \log 2-\frac{1}{6} \int_{\rho_{1}}^{r} t^{\delta} d t+\log m\left(\rho_{1}\right)
$$

Thus if $\delta>1$ then $\log \mu(r) \leq C r^{(1+\delta) / 2}-c r^{1+\delta}$, and if $0<\delta \leq 1$ then $\log \mu(r) \leq C r-c r^{1+\delta}$, where $C$ and $c$ are positive constants. Therefore, since $\delta>0$, for $r$ sufficiently large we have

$$
0<\mu(r) \leq C \exp \left(-\beta r^{1+\delta}\right)
$$

for some $\beta>0$. The functions $V$ and $\Psi$ defined above can easily be extended to $\mathbf{R}^{2}$ in a way that Theorem 2 is satisfied.

Proof of Lemma 3.1. We will smoothly modify in four steps the function $u_{1}=r^{-n} \exp (-i n \theta)$ into a function $u$ on $A(0,6)$ that satisfies (a), (b), (c), and (d). In this proof $C$ denotes a positive constant independent of $\rho, k$, and $n$.

I. The annulus $A(0,2)$. For $m=0,1, \ldots, 2 n+2 k-1$ we set $\theta_{m}=m T$, where $T \equiv \pi /(n+k)$. Let $f$ be a smooth $T$-periodic function on $\mathbf{R}$ such that $\int_{0}^{T} f(\theta) d \theta=0$, $f(\theta)=-4 k$ on $[0, T / 5] \cup[4 T / 5, T]$, and $-4 k \leq f(\theta) \leq 5 k$ and $\left|f^{\prime}(\theta)\right| \leq C k / T$, for $0 \leq \theta \leq T$. Set

$$
\Phi(\theta)=\int_{0}^{0} f(t) d t
$$

Clearly $\Phi$ is $T$ - and $2 \pi$-periodic, and $\Phi\left(\theta_{m}\right)=0$. In addition, for $\theta \in \mathbf{R}$ we have

$$
|\Phi(\theta)| \leq 5 k /(n+k), \quad\left|\Phi^{\prime}(\theta)\right| \leq 5 k, \quad \text { and } \quad\left|\Phi^{\prime \prime}(\theta)\right| \leq C k n
$$

and

$$
\Phi(\theta)=-4 k\left(\theta-\theta_{m}\right) \equiv-4 k \theta+b_{m}, \quad \text { for }\left|\theta-\theta_{m}\right| \leq T / 5
$$

Set $F(\theta)=(n+2 k) \theta+\Phi(\theta), b=\left(\rho+\rho^{(1-\delta) / 2}\right)^{-2 k}$, and $u_{2}=-b r^{-n+2 k} \exp (i F(\theta))$. Note that $\left|u_{1}(r, \theta)\right|=\left|u_{2}(r, \theta)\right|$ for $r=\rho+\rho^{(1-\delta) / 2}$; in addition, it follows from (3.4) that $u_{2}=-b r^{-(n-2 k)} \exp \left(i(n-2 k) \theta+i b_{m}\right)$ on the sectors

$$
S_{m} \equiv\left\{(r, \theta):\left|\theta-\theta_{m}\right| \leq T / 5\right\}, \quad m=0,1, \ldots, 2 n+2 k-1 .
$$

On $A(0,1 / 3)$ we have

$$
\begin{aligned}
\frac{\left|u_{2}(r, \theta)\right|}{\left|u_{1}(r, \theta)\right|} & =\frac{r^{2 k}}{\left(\rho+\rho^{(1-\delta) / 2}\right)^{2 k}} \\
& \leq\left(1+\frac{2}{3} \frac{1}{\rho^{(1+\delta) / 2}+\frac{1}{3}}\right)^{-2 k}
\end{aligned}
$$


Hence using the assumptions on $k$ and $\rho$ we obtain

$$
\left|u_{2}(r, \theta)\right| \leq \exp (-8)\left|u_{1}(r, \theta)\right| \quad \text { on } A(0,1 / 3) \text {. }
$$

Similarly

$$
\left|u_{2}(r, \theta)\right| \geq \exp (8)\left|u_{1}(r, \theta)\right| \quad \text { on } A(5 / 3,2)
$$

Let $\psi_{i}(r), i=1,2$, be $C^{\infty}$-functions taking values between 0 and 1 such that $\psi_{1}$ vanishes for $r \geq \rho+1.9 \rho^{(1-\delta) / 2}$ and equals 1 for $r \leq \rho+(5 / 3) \rho^{(1-\delta) / 2}, \psi_{2}$ vanishes for $r \leq \rho+0.1 \rho^{(1-\delta) / 2}$ and equals 1 for $r \geq \rho+(1 / 3) \rho^{(1-\delta) / 2}$, and

$$
\left|\psi_{i}^{(p)}(r)\right| \leq C \rho^{-p(1-\delta) / 2}, \quad r \geq 0 ; \quad i=1,2 ; p=1,2
$$

Define $u=\psi_{1} u_{1}+\psi_{2} u_{2}$. Clearly $u$ is harmonic in $S \equiv A(1 / 3,5 / 3) \cap\left(U S_{m}\right)$. Now set

$$
V(r, \theta)= \begin{cases}0 & (r, \theta) \in S \\ \Delta u / u & \text { otherwise }\end{cases}
$$

Clearly (3.1) holds on $A(0,2)$. Next we show that $|u|>0$ on $A(0,2) \backslash S$ and that (3.2) holds on $A(0,2)$.

On $A(0,1 / 3)$ we have

$$
\Delta u=\psi_{2} \Delta u_{2}+2 \psi_{2}^{\prime} \partial_{r} u_{2}+\left(\psi_{2}^{\prime} / r+\psi_{2}^{\prime \prime}\right) u_{2}
$$

and, using (3.5),

$$
|u| \geq\left|u_{1}\right|-\left|u_{2}\right| \geq \frac{1}{2}\left|u_{1}\right| \geq \exp (7)\left|u_{2}\right|>0
$$

Similarly on $A(5 / 3,2)$ we have

$$
\Delta u=\Delta u_{2}+2 \psi_{1}^{\prime} \partial_{r} u_{1}+\left(\psi_{1}^{\prime} / r+\psi_{1}^{\prime \prime}\right) u_{1}
$$

and, using (3.6),

$$
|u| \geq\left|u_{2}\right|-\left|u_{1}\right| \geq \exp (7)\left|u_{1}\right|>0
$$

A short calculation shows that on $A(0,2)$ we have

$$
\Delta u_{2}=\left[\frac{\left(4 k+2 n+\Phi^{\prime}\right) \Phi^{\prime}-8 k n}{r^{2}}+\frac{i \Phi^{\prime \prime}}{r^{2}}\right] u_{2}
$$

Using (3.3) we obtain 


$$
\left|\Delta u_{2}\right| \leq \frac{C k n}{r^{2}}\left|u_{2}\right|
$$

Thus, by the assumptions on $k, n, \epsilon$, and $\delta$, we have

$$
\left|\Delta u_{2}\right| \leq C r^{-c}\left|u_{2}\right| \quad \text { on } A(0,2)
$$

We also have

$$
\left|\psi_{2}^{\prime} \partial_{r} u_{2}\right|=\left|\psi_{2}^{\prime} \frac{n-2 k}{r} u_{2}\right| \leq C \frac{n}{r} \rho^{-(1-\delta) / 2}\left|u_{2}\right| \leq C r^{-c}\left|u_{2}\right|
$$

and

$$
\left|\left(\psi_{2}^{\prime} / r+\psi_{2}^{\prime \prime}\right) u_{2}\right| \leq C\left(\rho^{-(1-\delta) / 2} / r+\rho^{-(1-\delta)}\right)\left|u_{2}\right| \leq C r^{-\epsilon}\left|u_{2}\right|
$$

Combining these three last estimates, (3.8), and (3.9) we find that (3.2) holds on $A(0,1 / 3)$. Analogously, using (3.10), (3.11), and (3.12), we have that (3.2) holds on $A(5 / 3,2)$. It remains to show that $|u|>0$ and that $(3.2)$ holds on

$$
P_{m}=\left\{(r, \theta): \theta_{m}+\frac{T}{5} \leq \theta \leq \theta_{m}+\frac{4 T}{5}\right\} \cap A(1 / 3,5 / 3), \quad m=0, \ldots, 2 n+2 k-1 .
$$

For this purpose we set $G(\theta)=F(\theta)+n \theta$. On the annular sectors $P_{m}$ we have

$$
|u|=\left|u_{1}+u_{2}\right|=\left|u_{2}\right|\left|\exp (i G(\theta))-\frac{1}{b r^{2 k}}\right|
$$

We will show now that for some $\eta>0$ we have

$$
\left|\exp (i G(\theta))-\frac{1}{b r^{2 k}}\right| \geq \eta, \quad(r, \theta) \in P_{m}, \quad m=0, \ldots, 2 n+2 k-1 .
$$

Using this last, (3.12), and the fact that $\Delta u=\Delta u_{2}$ on $P_{m}$ we obtain $|u|>\eta\left|u_{2}\right|$ and therefore (3.2) holds on $P_{m}$. To prove (3.14) note that $G(\theta)=2(n+k) \theta+\Phi(\theta)$ and $G^{\prime}(\theta)=2(n+k)+f(\theta)$. Hence by the assumptions of $f, k$, and $n$ we may assume that $G^{\prime}(\theta)>n>0$. Since $G\left(\theta_{m}\right)=2 \pi m$ and $G\left(\theta_{m+1}\right)=2 \pi(m+1)$ we conclude that

$$
2 \pi m+\frac{n T}{5} \leq G(\theta) \leq 2 \pi(m+1)-\frac{n T}{5} \quad \text { for } \quad \theta_{m}+\frac{T}{5} \leq \theta \leq \theta_{m}+\frac{4 T}{5} .
$$

Using the definition of $T$ and the assumptions on $k$ and $n$ we find that

$$
2 \pi m+\frac{\pi}{7} \leq G(\theta) \leq 2 \pi(m+1)-\frac{\pi}{7} \quad \text { for } \quad \theta_{m}+\frac{T}{5} \leq \theta \leq \theta_{m}+\frac{4 T}{5} .
$$


It follows from this last estimate and (3.13) that (3.14) holds with $\eta=\sin (\pi / 7)$.

II. On $A(2,3)$ we deform $u_{2}$ into $u_{3}=-b r^{-n+2 k} \exp i(-n+2 k) \theta$. Let $\psi(r)$ be a $C^{\infty}$ function which takes values between 0 and 1 , equals 1 for $r \leq \rho+(7 / 3) \rho^{(1-\delta) / 2}$, vanishes for $r \geq \rho+(8 / 3) \rho^{(1-\delta) / 2}$, and satisfies (3.7). On $A(2,3)$ we set $u=-b r^{-n+2 k} \exp i(\psi(r) \Phi(\theta)$ $+(n+2 k) \theta)$ and $V=\Delta u / u$. A short calculation shows that

$$
\Delta u=\left[\left(\frac{-n+2 k}{r}+i \psi^{\prime} \Phi\right)^{2}+i \Phi\left(\frac{\psi^{\prime}}{r}+\psi^{\prime \prime}\right)-\frac{\left(n+2 k+\psi \Phi^{\prime}\right)^{2}}{r^{2}}+\frac{i \psi \Phi^{\prime \prime}}{r^{2}}\right] u
$$

Using (3.3), the assumptions on $\psi^{(p)}$, and the assumptions on $k, n, \epsilon$, and $\delta$ we find that $\psi^{\prime} \Phi=O(1 / r)$, that $\psi \Phi^{\prime}=O(k)$, that $\psi \Phi^{\prime \prime}=O(k n)$, and that $\Phi\left(\psi^{\prime} / r+\psi^{\prime \prime}\right)=O\left(r^{-c}\right)$. Hence

$$
\Delta u=\left[\frac{O(n k)}{r^{2}}+O\left(r^{-\epsilon}\right)\right] u .
$$

Using again the assumptions on $k, n, \epsilon$, and $\delta$ we find that (3.2) holds on $A(2,3)$.

III. On $A(3,4)$ we deform $u_{3}$ into $u_{4}=-b r^{-(n+2 k)} \exp i(n+2 k) \theta$, where $b$ is as in I and $d \equiv\left(\rho+3 \rho^{(1-\delta) / 2}\right)^{4 k}$. Let $\psi$ be a $C^{\infty}$-function which takes values between 0 and 1 , equals 1 for $r \leq \rho+(10 / 3) \rho^{(1-\delta) / 2}$, vanishes for $r \geq \rho+(11 / 3) \rho^{(1-\delta) / 2}$, and satisfies (3.7). Next we define $h(r)=\psi(r)+(1-\psi(r)) d r^{-4 k}$. It is easily verified using the assumptions on $\psi, k$, and $\delta$ that $h$ satisfies (3.7) and that

$$
h(r) \geq d r^{-4 k} \geq\left(1+\frac{1}{\rho^{(1+\delta) / 2}+1}\right)^{-4 k} \geq \exp (-25(1+\delta)) .
$$

Now we set $u=u_{3} h$ and $V=\Delta u / u$, and verify as above that (3.2) holds on $A(3,4)$. In addition, on $A(11 / 2,4)$ we have $u=-b d r^{-(n+2 k)} \exp i(n+2 k) \theta$.

IV. Finally on $A(4,6)$ we deform $u_{4}$ into $u_{5}=a r^{-n-k} \exp i(-n-k) \theta$, where $a \equiv b d\left(\rho+5 \rho^{(1-\delta) / 2}\right)^{-k}$ and $b$ and $d$ are as in III. Note that $a$ has been chosen so that $\left|u_{4}(r, \theta)\right|=\left|u_{5}(r, \theta)\right|$ for $r=\rho+5 \rho^{(1-\delta) / 2}$. Let $\psi_{i}(r)$, $i=1,2$, be $C^{\infty}$-functions taking values between 0 and 1 and satisfying (3.7), such that $\psi_{1}$ vanishes for $r \geq \rho+5.9 \rho^{(1-\delta) / 2}$ and equals 1 for $r \leq \rho+(17 / 3) \rho^{(1-\delta) / 2}$, and $\psi_{2}$ vanishes for $r \leq \rho+4.1 \rho^{(1-\delta) / 2}$ and equals 1 for $r \geq \rho+(13 / 3) \rho^{(1-\delta) / 2}$. Now on $A(4,6)$ we set $u=\psi_{1} u_{4}+\psi_{2} u_{5}$. It is clear that $u$ is harmonic on $A(13 / 3,17 / 3)$. Therefore we set $V=0$ on this annulus. We verify as in I that $V=\Delta u / u$ satisfies (3.2) on the remaining points of $A(4,6)$.

To finish this proof we set $m(r)=\max \{|u(r, \theta)|, 0 \leq \theta \leq 2 p\}$ and

$$
M(r)= \begin{cases}r^{-n} & \rho \leq r \leq \rho+\rho^{(1-\delta) / 2} \\ b r^{-n+2 k} & \rho+\rho^{(1-\delta) / 2} \leq r \leq \rho+3 \rho^{(1-\delta) / 2} \\ b r^{-n+2 k} h(r) & \rho+3 \rho^{(1-\delta) / 2} \leq r \leq \rho+4 \rho^{(1-\delta) / 2} \\ b d r^{-n-2 k} & \rho+4 \rho^{(1-\delta) / 2} \leq r \leq \rho+5 \rho^{(1-\delta) / 2} \\ a r^{-n-k} & \rho+5 \rho^{(1-\delta) / 2} \leq r \leq \rho+6 \rho^{(1-\delta) / 2}\end{cases}
$$


where $a, b$, and $d$ are as in IV. It is clear that $M(r)$ is a continuous piecewise smooth function on $\left[\rho, \rho+6 \rho^{(1-\delta) / 2}\right]$ that satisfies $m(r) \leq 2 M(r), m(\rho)=M(\rho)$, and

$$
\frac{d}{d r} \log M(r)=\frac{-n+O(k)}{r} \leq-\frac{\rho^{1+\delta}}{2 r} \leq-\frac{1}{6}\left(\rho+6 \rho^{(1-\delta) / 2}\right)^{\delta} \leq-\frac{1}{6} r^{\delta} .
$$

Therefore

$$
\log m(r)-\log m(\rho) \leq \log 2+\int_{\rho} \frac{d}{d r} \log M(t) d t \leq \log 2-\frac{1}{6} \int_{\delta}^{r} d t
$$

which proves Lemma 3.1 .

Acknowledgements. I would like to thank Professor I. Herbst for suggesting this problem as well as Meshkov's paper to me, and for very helpful conversations. I would also like to thank Professor L. Hörmander for useful comments.

\section{REFERENCES}

1. S. Agmon, Lectures on Exponential Decay of Solutions of Second-Order Elliptic Equations. Bounds on Eigenfunctions of $\mathrm{N}$-body Schrödinger Operators, Mathematical Notes 29, Princeton University Press, 1982.

2. S. Agmon, On the Asymptotic Behavior of Solutions of Schrödinger Equations in Unbounded Domains, Analyse de Mathématique et Applications, Gautier-Villars, Paris, 1988.

3. R. Carmona and B. Simon, Pointwise bounds on eigenfunctions and wave packets in $\mathrm{N}$-body quantum systems, V: lower bounds and path integrals, Comm. Math. Phys. 80 (1981), 59-98.

4. J. CRUz, Boundary Values at Infinity of Solutions to the Schrödinger Equation, Ph.D. Thesis, University of Virginia, 1991.

5. P. Deift, W. Hunziker, B. Simon and E. Vock, Pointwise bounds on eigenfunctions and wave packets in $N$-body quantum systems IV, Comm. Math. Phys. 64 (1978), 1-34.

6. R. Froese and I. HerbST, Exponential lower bounds to solutions of the Schrödinger equation: lower bounds for the spherical average, Comm. Math. Phys. 92 (1983), 71-80.

7. R. Froese, et al., $L^{2}$-Exponential lower bounds to solutions of the Schrödinger equation, Comm. Math. Phys. 87 (1982), 265-286.

8. R. Froese, et al., $L^{2}$-Lower bounds to solutions of one-body Schrödinger equations, Proc. Roy. Soc. Edinburgh 95A (1983), 25-38.

9. L. Hörmander, The Analysis of Linear Partial Differential Operators, Vol. II, (Springer Verlag, 1985).

10. V. MeshKov, On the possible rate of decay at infinity of solutions of second-order partial differential equations, Math. U.S.S.R.-Sb. 72 (1992), No. 2.

11. J. SAUt and B. SCheurer, Remarques sur un théoreme de prolongament unique de Mizohata, C. R. Acad. Sc. Paris 296 (1983), 307-310. 
12. B. Simon, Schrödinger semigroups, Bull. Amer. Math. Soc. 7 (1982), 447-526.

\section{Departamento de Matemáticas}

UNIVERSIDAD DE LAS AMÉricas-PuEbla

Cholula, Pue. 72820

MeXICO

and

Department of Mathematics

UNIVERSITY OF VIRGINIA

Charlottesville, VA 22903

U.S.A 